\title{
Comparative Analysis of Health Behaviors, Health Status, and Medical Needs among One-Person and Multi- Person Household Groups: Focused on the Ageing Population of 60 or More
}

\author{
Su-Jung Lee', Sun-Hee Lee ${ }^{2 * *}$ \\ 'Department of Medical Science, Ewha Womans University Graduate School, Seoul, Korea \\ ${ }^{2}$ Department of Preventive Medicine, Ewha Womans University College of Medicine, Seoul, Korea
}

\begin{abstract}
Background: Among the issues caused by the increase of one-person households the health problems and primary medical needs of one-person elderly households among the issues with the increase in one-person households is very important. The purpose of this study was to identified health care needs and problems of one-person elderly households by comparing health behavior, health status, disease prevalence rate, and medical needs to one-person elderly households and multi-person households.

Methods: Data were obtained from the 2017 Community Health Survey. In this study, 66,211 people who were over 60 years of age without missing variables were included in the final analysis.

Results: One-person households showed poorer overall health behaviors, such as nutritional behaviors, exercise behaviors, drinking and smoking behaviors, and poorer subjective and objective health status than multi-person households. Furthermore, albeit the high requirement of medical services for mental health outcomes such as suicide attempts, the rate of not receiving services was also high indicating high barriers to receive and access health care.

Conclusion: Comprehensive and direct comparison of health and medical factors revealed the medical vulnerability and unmet needs in terms of health promotion in the single-person elderly households. These results can be applied as the basic information to understand the needs and medical problems of these risk groups by primary medical doctors who predominantly treat single-person elderly households and can also be used as basic data for policy development in order to solve these unmet medical care needs.
\end{abstract}

Keywords: Family Characteristics; Health Behavior; Health Status; Medical Need

Received: February 22, 2020, Revised: May 15, 2020, Accepted: May 20, 2020

${ }^{*}$ Corresponding Author: Sun-Hee Lee https://orcid.org/0000-0002-3628-7627

Tel: +82-2-6986-6249, E-mail: Ish0270@ewha.ac.kr 


\section{INTRODUCTION}

The family structure in Korea has changed from the traditional large family center to the nuclear family form due to industrialization and urbanization, and recently, the number of one-person households living alone, and the nuclear family has increased rapidly. ${ }^{1)}$ According to the National Statistical Office, more than one-third of Korea's population is estimated to be one-person households: $15.5 \%$ in $2000,29.3 \%$ in 2018, and $34.3 \%$ in 2035. ${ }^{2)}$

The increase in one-person households is an inevitable social phenomenon caused by changes in social structure such as industrial reorganization and changes in values. Yet as social security and tax, housing, and consumer markets are built around general households, one-person households are excluded or disadvantaged and one-person households are more likely to be at greater risk than ordinary households in various areas of life, including fear of aging and safety. ${ }^{3)}$

Along with the increase in the number of one-person households, the rapid increase in the number of elderly one-person households due to the growing age levels has been a notable phenomenon. In 1994 , the proportion of the elderly living alone was $13.6 \%$, while in 2018 it was $19.4 \%$, an increase of about $6 \%$ across 18 years. ${ }^{4)}$ Compared to other elderly groups, one-person households have a problem in that they need to maintain their own lives independently because there is no family to support them. It was reported that one-person elderly households were more likely to be threatened by serious psychological and social problems due to financial problems, mental health problems such as depression, low nutrition and physical health, and lack of health promotion. ${ }^{5,6)}$ Especially, among the one-person households, the need for medical care is expected to be an important issue compared to other age groups, as the elderly group's lower socioeconomic status is combined with their health problems. The problem of increasing elderly medical expenses is that $14 \%$ of the elderly still spend $40 \%$ of their health insurance expenditures, and are expected to have the highest expenditure causing social and pension burden in the future in an aged society. ${ }^{7)}$ Among the issues caused by the increase of one-person households due to social change, the provision of measures for health problems and primary medical needs of one-person elderly households is the most important policy issue to be addressed in the future.

Thus far, diverse studies have been conducted due to the increasing social interest in one-person households. Although there are many studies focusing on socio-demographic factors, ${ }^{3)}$ nutritional behavior, ${ }^{8,9)}$ quality of life, ${ }^{5,10)}$ and satisfaction, ${ }^{6}$ there are only few studies focusing on health factors. Such studies only conducted short analysis for general health status, ${ }^{1,11,12)}$ mental health, ${ }^{13-18)}$ and oral health status. In addition, since only one one-person elderly household is analyzed, there are not many studies comparing the characteristics of one-person and multi-person households. Most studies involving two groups did not use comparison models between groups ${ }^{11,12,14,17)}$ and, thereby, the differences between the two groups have not been systematically investigated. Therefore, relevant studies are urgent as the problems- based alternatives can be sought only after in-depth understanding of the health problems and medical needs of one-person elderly households.

This study aimed to identify the health and primary medical needs problems of one-person elderly households by using the 2017 Community Health Survey data. Specifically, the health needs and problems of one-person elderly households and multi-person households were compared by analyzing the health behavior, health status, disease prevalence, medical needs, and medical access to the identified health care needs and problems of one-person elderly households.

\section{METHODS}

\section{Study Population}

This is a cross-sectional study using raw data from the 2017 Community Health Survey. Community health surveys have been undertaken since 2008 in accordance with Article 4 of the Local Health Act (Contents of the Regional Health Care Plan) to lay the groundwork for local health projects based on scientific evidence. The 2017 Community Health Survey was conducted for about 3 months, from August 16, 2017 to October 31, 2017, where a trained surveyor visited selected sample households. The data were collected through a one-on-one interview with a laptop equipped with a survey program. ${ }^{19}$

The total survey targets of the 2017 Community Health Survey were 228,381 people, aged 19 or older who were extracted under the complex sample design which stratified the dong/eup/myeon housing types and stratified the samples. In this study, people who were over 60 years of age were selected for analysis. In community health surveys, the unit of survey is each household; every household member responds to the common questionnaires (e.g., health status, health behavior, smoking and drinking habits), while only the representative household member responds to questionnaires on household characteristics (e.g., types of households, whether recipient of national basic livelihood guarantees or not, etc.). ${ }^{19)}$

Accordingly, 66,211 people over 60 years of age without any missing data for relevant variables were selected as the final analysis target among the sample of the 2017 Community Health Survey. This study was approved by the Institutional Review Board of the Seoul Ewha Womans University Medical Center, Korea (protocol no., SEUMC 202004009-HE001).

\section{Definition of Variables}

\section{1) Household form}

In this study, interview survey data from household types were used to compare single-person households and multi-person household groups. Single-person households were identified from the community health survey data as subjects who responded as single-person households without other household members, and multi-person households were identified as all other households (1st, 2nd, 3rd generation). 


\section{2) Demographic characteristics}

The socio-demographic characteristics of this study used interview questionnaire data on gender, age, household income, and education level. Gender was categorized as male and female, and age was used as a continuous value based on age. For household income, raw data of household average monthly income on an 8-point scale were used. The level of education was divided into three groups: graduated elementary school or lower, graduated middle school, and graduated high school or higher.

\section{3) Health behavior}

As a result of selecting the variable groups that can be classified as health behavior in the interview data, the items of lifelong smoking and lifelong drinking were used. We defined smoking and drinking status based on the original multiple-choice questionnaire of the community health survey data; smoking was classified as "non-smoking for life," and smoking over five packs (100 cigarettes) was classified as "smoking." Drinking was classified as "lifetime non-drinking" and "drinking" if the respondent ever drank more than one drink.

Interview questions on exercise and physical activity were used as exercise behaviors, and flexible exercise days and muscular exercise days were classified into two groups, "yes" for "1-5 days or more," and "no" for "none at all."

For nutritional form, interview questions on eating habits were used, and salt intake level and nutrition indicator recognition were included. The salt intake levels were divided into two groups: "eat salty," "eat lightly." Nutrition indicator recognition was classified as "yes" or "no."

\section{4) Health status}

(1) Subjective health status

Subjective health status was used for subjective health level, subjective stress level, subjective oral health level, depression experience, and sleep time. Subjective health levels and subjective oral health levels were classified into three groups: "bad," "normal," and "good." Subjective stress levels were classified into three groups: "a lot", "little" and "hardly." The original categories for "yes" and "no" from the raw data were used to categorize depression status. Sleep time was a continuous hour value based on the original data.

\section{(2) Disease status}

The disease status was based on the experience of being diagnosed with four disease types including hypertension, diabetes, arthritis, and cataract, and the original multiple-choice questions divided into "yes" and "no" were used to categorize disease status. Each of the four diseases was coded as 1 when there was a disease, and in regression analysis, four disease values were summed and used to avoid the complexity of the regression model. The higher the disease combined score, the more likely a person has experienced target diseases.

\section{5) Necessity of mental health checks}

Medical needs were identified with suicide experience and suicide attempt experience. If there was such an experience, mental health needs were determined. The total scores of suicide experiences and suicide attempts are summarized as mental health variables. Higher scores indicate suicide experience and suicide attempt experience.

\section{6) Oral health examination not received}

The oral health examination included the annual dental care examination and the annual oral examination. Both variables were classified as "yes", and "no." The oral care approach used in the regression analysis was used by adding the number scores coded in the same direction of the abovementioned variables. Higher scores indicate lower oral examinations.

\section{7) Medical care not received}

Not receiving medical service examinations included inoculation, regular health examination, cancer examination, and necessary medical care unexanmination and were all analyzed in binary variable form. The inoculation and examination approach used in the regression analysis was coded to represent the same meaning, and the scores of each variable were summed; the higher the score, the lower the number of medical services received.

\section{Statistical Analysis}

Since the sample from the Community Health Survey was extracted under the complex sample design rather than the simple random sample design, the complex sample analysis method is recommended. Therefore, this study analyzed the stratification variables, cluster variables, and weights of the complex sample design survey factors based on the analysis guidelines of the Community Health Survey. ${ }^{20)}$ All data were analyzed using IBM SPSS ver. 24.0 (IBM Corp., Armonk, NY, USA). To compare and analyze the socio-demographic characteristics, health behaviors, disease prevalence, and medical needs of the one-person household and multi-person household, multiple sample chi-square test, t-test tests, and multiple sample logistic regression were performed. In all the analyses, statistical significance was found when the significance probability (P-value) was $<0.05$.

\section{RESULTS}

\section{Comparison of Socio-Demographic Characteristics between One-Person and Multi-Person Household Groups} Table 1 shows a comparison of socio-demographic characteristics between one-person and multi-person households. In terms of gender, the proportion of females in the one-person household was $74.3 \%$, which was significantly higher than $50.3 \%$ of multi-person households. The gender distribution between one-person households and multiperson households was so large that the differences due to gender could act as confounding variables. Therefore, for comparison of other characteristics, a comparative analysis was conducted by subdividing 


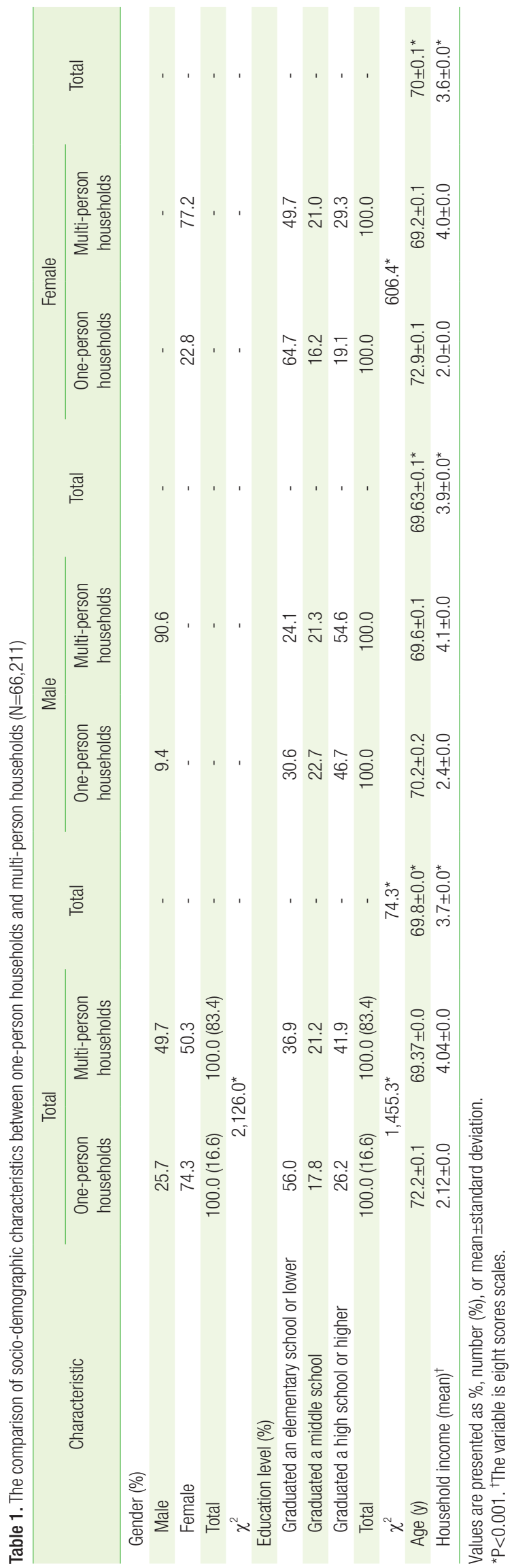

gender into subgroups.

In terms of average age, the one-person household group was 72.2 years old, which was significantly higher than the multi-person household group. In the subgroup analysis of male and female groups, the age of one-person households was higher than that of multi-person households $(\mathrm{P}<0.001)$. In terms of household income, the income level of one-person households was lower than that of multi-person households $(\mathrm{P}<0.001)$, and the same result was found in the gender subgroup analysis. For education level, one-person households were significantly lower than that of the multi-person households $(\mathrm{P}<0.001)$. Compared to multi-person households, one-person households had a relatively higher percentage of graduates from elementary school or lower $(56.0 \%)$ than graduates from a middle school (17.8\%), and graduates from a high school or higher (26.2\%). Similar results were also obtained when the analysis was divided into female and male $(\mathrm{P}<0.001)$.

\section{Comparison of Health Behavior and Health Status of One- Person and Multi-Person Households}

The results of comparing the health behaviors between the one-person and multi-person households are shown in Table 2. The ratio of smokers was $25.7 \%$ for one-person households and $39.8 \%$ for multiperson households. When divided into male and females, in the case of one-person households, $80.4 \%$ were men and $6.7 \%$ were women, thus one-person households had higher percentage of smokers than multi-person households $(\mathrm{P}<0.001)$. Drinking was $74.4 \%$ in multi-person households, which was higher than one-person households, and a similar result was found in the gender subgroup analysis. In terms of muscular exercise days (56.4\%) and flexible exercise days (87.8\%), larger proportion of one-person households answered "no," thus was significantly higher compared to multi-person households, and the gender sub-analysis result was the same. In general, the level of salt intake was higher in one-person households with $25.0 \%$ reporting "salty" than in multi-person households $(\mathrm{P}<0.05)$. The proportion of respondents who answered "no" for nutrient indicator recognition was $74.8 \%$ $(\mathrm{P}<0.001)$, thus higher for one-person households.

Table 3 compares the health status of the one-person and multi-person groups. Among the subjective health levels, the proportion of respondents who answered "bad" was $44.8 \%$ for one-person households and $31.5 \%$ for multi-person households, and the one-person households were significantly higher. Dividing into males and females, $36.8 \%$ of males and $47.6 \%$ of females answered "bad" in one-person households, higher compared to the multi-person households $(\mathrm{P}<0.001)$. Among the subjective stress levels, the $19.0 \%$ of one-person household respondents responded "a lot," and $18.0 \%$ of multi-person households, thus being significantly higher in one-person households $(\mathrm{P}<0.001)$. The percentage of respondents who answered "hardly" was $36.8 \%$ for one-person households, which was significantly higher than $31.8 \%$ for multi-person households. In the gender sub-analysis, males responded "a lot" at a rate of $21.1 \%$ for one-person households and $14.7 \%$ for multi-person households. Yet in the case of women, $18.3 \%$ of one-per- 


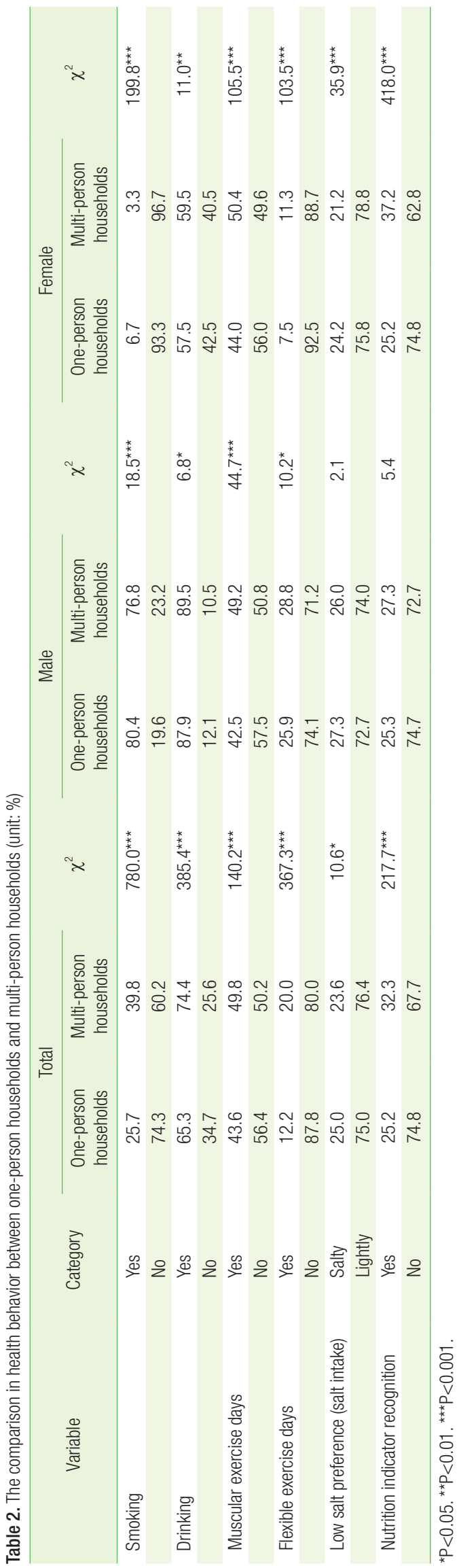

son households answered "a lot," thus it was slightly lower than the 21.3\% from multi-person households $(\mathrm{P}<0.001)$.

The subjective oral health level was "bad" for $57.5 \%$ of one-person households and $48.0 \%$ for multi-person households. The proportion of one-person households who said it was "bad" was thus significantly higher $(\mathrm{P}<0.001)$ to make it the same as the gender sub-analysis result. The rate of depression was $11.4 \%$ for one-person households and $6.4 \%$ for multi-person households, thus significantly higher $(\mathrm{P}<0.001)$ for one-person households to make it the same as gender sub-analysis result. In terms of sleep time, 6.2 hours reported by one-person households and 6.5 hours by multi-person households, made it significantly less than one-person households. In the gender sub-analysis, oneperson households for both males and females showed less sleep time than multi-person households. In the case of disease, the rate of diagnosed diseases was significantly higher in the one-person household than the multi-person, including $54.5 \%$ for hypertension, $22.6 \%$ for diabetes, $37.0 \%$ for arthritis, and $35.3 \%$ for cataracts. The gender subanalysis revealed that the proportion of disease in the one-person household was high $(\mathrm{P}<0.001)$. In men, the rate of cataracts was not significant.

\section{Comparison of Mental Health Service Needs and Medical Accessibility among One-Person and Multi-Person Household Groups}

Table 4 compares if there is not a problem in terms of the needs and accessibility of mental health services, oral and general health services. First, when asked about suicide experience and suicide attempt experience as an indicator of the need for mental health service, $15.1 \%$ of one-person households reported suicide experience and $0.7 \%$ experienced suicide attempt, these were significantly higher than multi-person households, and the same result was found in the sub-analysis of male and female classification.

If they did not receive oral examination, health examination, or necessary medical services, they found that there was a problem with access to medical services. The questionnaire on these service items showed that $71.1 \%$ of one-person households did not receive oral examination, $19.8 \%$ did not receive necessary dental care, $27.0 \%$ did not receive regular health examinations, $11.9 \%$ said they did not receive cancer screening. These were higher than multi-person households, indicating that access to necessary medical services was relatively inferior. The same result was found in the sub-group analysis by gender. However, in the case of vaccinations, the ratio of "not received" was higher in multi-person households than in one-person households.

\section{Logistic Analysis to Compare Health and Medical Characteristics of One-Person Household and Multi- Person Household Groups}

Health behavior, health status, and service use level were all independent variables. The results of the logistic regression analysis to identify the differences between one-person households and multi-person households are shown in Table 5. When the age, income level, and 


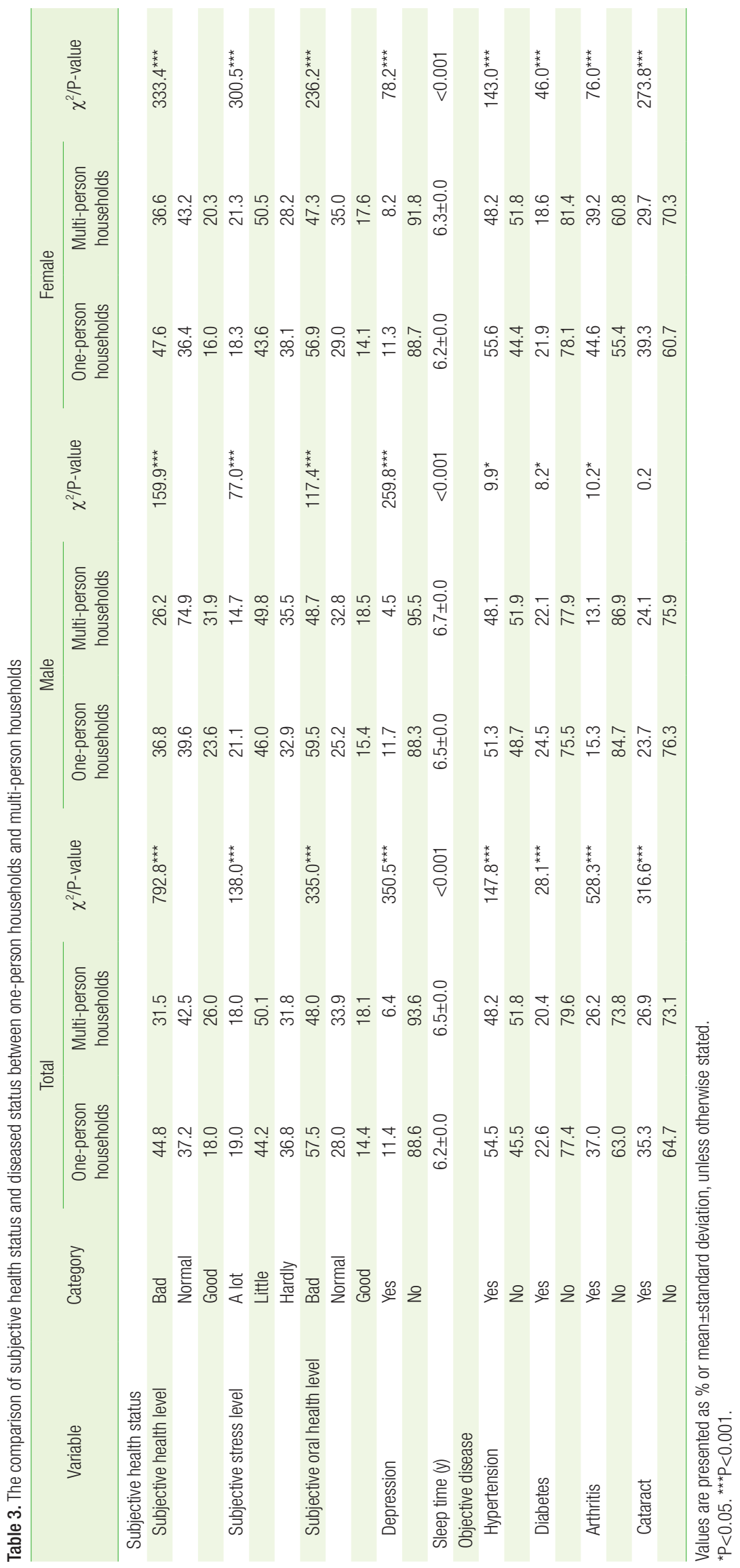




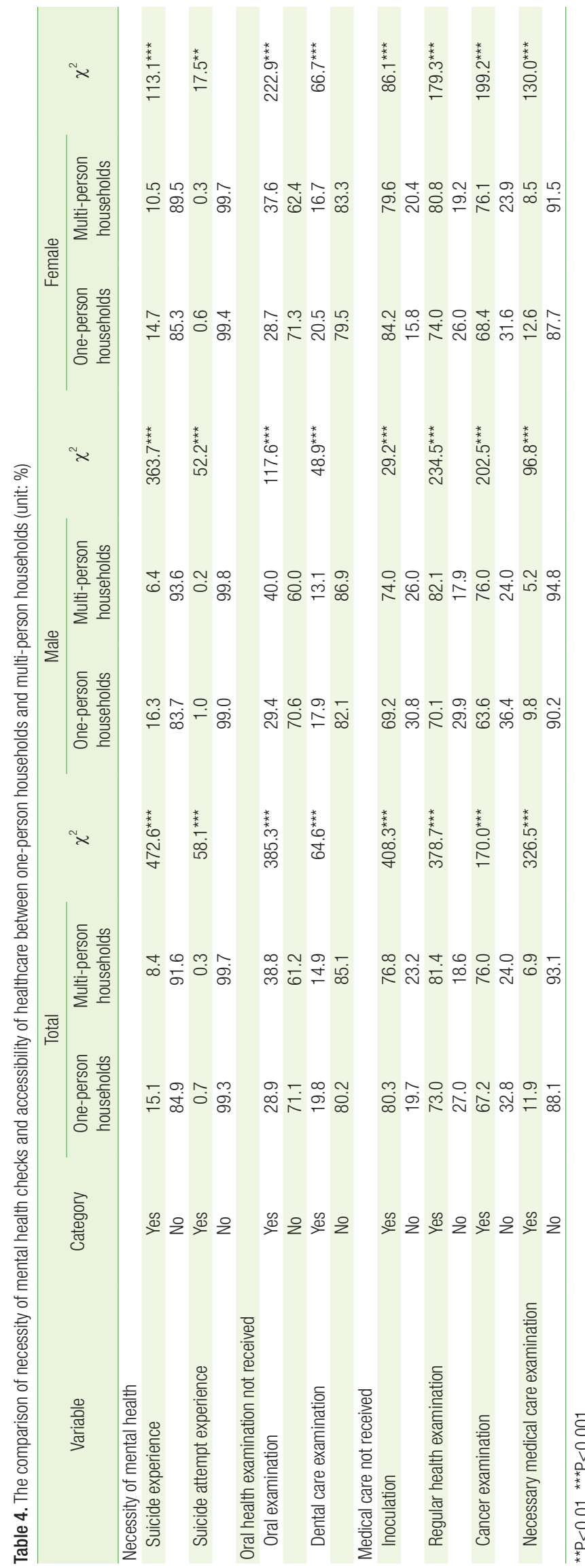

household income were controlled, as a "nutrition behavior" factor, the one-person household group that eats "salty" compared to "blandly" is 0.94 times lower than the multi-person household group. On the other hand, the status of "perception" of nutrition labeling is 1.55 times higher than that of "not perception," thus the nutritional behavior of one-person households can be judged to be rather bad. In addition, one-person households had significantly higher lifetime smoking or lifetime drinking than multi-person households, and their sleep time was significantly lower (0.92 times). In terms of exercise behavior, the number of daily strength exercise and flexibility exercise days was also significantly lower than that of multi-person households.

Sleeping time was less in one-person households than in multi-person households, and subjective health was significantly worse in "awareness of health" and "awareness of oral health," and a higher awareness of depression. "Awareness of stress level" was significantly lower. If diagnosed as a disease, the medical needs of one-person households were significantly higher than the multi-person households. Mental health service needs, including suicide experience, were also significantly higher among one-person households. It was confirmed that the medical needs of single household group were high. Furthermore, the oral related needs service non-reception level and health examination as well as medical use service non-reception level was also significantly higher for one-person households than for multiperson households.

\section{DISCUSSION}

In order to meet the policy demands of increasing social interest in one-person households due to the rapid increase in one-person households, this study investigated the health and medical needs of one-person elderly households, which are health-vulnerable groups. For this, we compared and analyzed the health behaviors, health status, and medical needs of one-person and multi-person households. About $16.6 \%$ of the total analysis subjects aged 60 or older were oneperson households and compared to the statistics reported in 2018 where $19 \%$ of the elderly aged 65 were one-person households, it was assessed to be at a similar level as the nationwide statistics. ${ }^{18)}$

As a result of comparing socio-demographic characteristics of oneperson and multi-person households, the proportion of females in one-person households was $74.3 \%$, higher than that in multi-person households (50.3\%). The higher trend of females in one-person households compared to multi-person households shows the same results in all studies involving one-person households. ${ }^{10,11,21)}$ The reason for this is that husbands are generally older than their wives and females have a longer average life span than males. Therefore, the gender ratio of the elderly population is thought to be due to the trend of decreasing men and increasing women, which may be affected by the difference in remarriage rates. ${ }^{5)}$ However, this large difference in gender characteristics may affect the comparison of other characteristics, which is a confounding variable. Therefore, when comparing household characteristics, sub-analysis was conducted by dividing into male 
Table 5. The result of the logistic regression to the difference between one-person households and multi-person households

\begin{tabular}{|c|c|c|c|}
\hline \multirow{2}{*}{ Variable } & \multicolumn{2}{|c|}{ One-person households ${ }^{\dagger}$} & \multirow{2}{*}{ P-value } \\
\hline & B & Adjusted odds ratio (95\% confidence interval) & \\
\hline Age & 0.02 & $1.02(1.01-1.02)$ & $<0.001^{\star \star \star}$ \\
\hline Gender (ref: male) & 1.27 & $3.56(3.25-3.91)$ & $<0.001^{\star \star *}$ \\
\hline Household income & -0.88 & $0.42(0.41-0.43)$ & $<0.001^{\star \star \star}$ \\
\hline Low salt preference (salt intake) (ref: salty) & -0.07 & $0.94(0.90-0.98)$ & $<0.01^{\star \star}$ \\
\hline Nutrition indicator recognition (ref: yes) & 0.44 & $1.55(1.48-1.62)$ & $<0.001^{\star \star \star}$ \\
\hline Smoking (ref: no) & 0.34 & $1.40(1.30-1.51)$ & $<0.001^{\star * *}$ \\
\hline Drinking (ref: no) & 0.12 & $1.12(1.07-1.18)$ & $<0.001^{\star * *}$ \\
\hline Muscular exercise days (ref: no) & -0.68 & $0.93(0.90-0.97)$ & $<0.001^{\star \star \star}$ \\
\hline Flexible exercise days (ref: no) & -0.56 & $0.57(0.54-0.61)$ & $<0.001^{\star \star \star}$ \\
\hline Subjective health level (ref: bad) & -0.50 & $0.61(0.58-0.63)$ & $<0.001^{\star * *}$ \\
\hline Subjective stress level (ref: a lot) & 0.45 & $1.57(1.51-1.64)$ & $<0.001^{\star \star \star}$ \\
\hline Subjective oral health level (ref: bad) & -0.23 & $0.79(0.76-0.82)$ & $<0.001^{\star \star *}$ \\
\hline Depression (ref: no) & 0.54 & $1.71(1.60-1.84)$ & $<0.001^{\star \star \star}$ \\
\hline Sleep time & -0.08 & $0.92(0.91-0.94)$ & $<0.001^{\star * *}$ \\
\hline Combined disease score ${ }^{\ddagger}$ & 0.04 & $1.04(1.01-1.07)$ & $<0.01^{\star \star}$ \\
\hline Necessity of mental health ${ }^{\S}$ & 0.21 & $1.24(1.14-1.34)$ & $<0.001^{\star \star \star}$ \\
\hline No having oral health examination" & 0.03 & $1.03(1.00-1.07)$ & $<0.05^{\star}$ \\
\hline No having medical careף & 0.16 & $1.17(1.14-1.20)$ & $<0.001^{\star * *}$ \\
\hline
\end{tabular}

Ref, reference.

${ }^{*} \mathrm{P}<0.05 .{ }^{* *} \mathrm{P}<0.01 .{ }^{* * *} \mathrm{P}<0.001 .{ }^{\dagger}$ Multi-person households are reference. ${ }^{\ddagger} \mathrm{C}$ Combined hypertension and diabetes and arthritis and cataract scores. ${ }^{\circledR} \mathrm{C}$ ombined suicide experience and suicide attempt experience scores. "Combined oral examination and dental care examination scores. "Combined inoculation and physical examination and cancer examination and necessary healthcare examination scores.

and female.

Age, which is a key socio-demographic variable, was significantly higher at the age of 72.2 for one-person household than multi-person households. In terms of household income, the one-person households had lower incomes, and lower educational levels with $56.0 \%$ of the students being elementary school graduates or below. These interfamily characteristics showed results in the same context as other studies related to one-person households ${ }^{1,11)}$ and showed the same trends when compared to men and women.

Regarding smoking, $25.7 \%$ of one-person households and $39.8 \%$ of multi-person households reported smoking, thus the percentage of smokers was significantly higher for multi-person households than one-person households. In the gender sub-analysis, the proportion of smokers in the one-person household was significantly higher than that of the multi-person household. In case of drinking, $74.4 \%$ of multiperson households drank, which was higher than one-person households, and the same result was found in gender sub-group analysis. This is consistent with the finding of $\mathrm{Kim}^{21)}$ that the elderly living with spouses drank more than those who did not live with spouses. In terms of "days of muscle exercise" and "days of flexibility exercise," the households that "never exercised" were significantly higher in the oneperson households than in the multi-person households. Kim ${ }^{21)}$ found similar results that those who exercised more than 3 times a week were more likely in the elderly who lived with their spouses compared to those who did not. Elderly people in Korea have predominantly spent their youth in a poverty-stricken society, and due to the lack of methods and facilities to enjoy leisure activities, they frequently spend their free time tediously without properly utilizing their leisure time. In the case of the elderly people living alone, there are differences in social interaction, exercise, and leisure activities participation forms depending on the presence of a spouse or cohabiting family. The level of active leisure participation varies according to economic and health conditions as well. Active leisure participation during old age can restore the self that was lost due to the losses of old age by forming a formal network of relationships and fulfilling its role. There is a need to work to increase participation in productive leisure activities in the sense that it is a major means of promoting physical and mental health. $^{22)}$

As an indicator of nutritional and dietary behavior, the "usual salt intake level" was slightly higher in one-person households than in multi-person households with those responding as "eating salty" higher and this is in agreement with the finding of Jang and Hong ${ }^{8)}$ about half the one-person households agreeing that "they usually eat spicy and salty foods." The ratio of eating spicy and salty foods is high. As a proxy for nutritional knowledge, the question of "perception of nutrition labeling" was shown to indicate that since the one-person household is lower than the multi-person household, the nutritional knowledge of the one-person household is somewhat lower than that of the multi-person household. Through this, the nutritional status of the elderly people living alone should be considered as a priority, and practical programs and health management support based on the health needs of the elderly people living alone should be constructed. ${ }^{23)}$

The proportion of one-person households that responded that the "subjective health level" was "bad" was high compared to the multiperson household group, and the "subjective stress level" and "depression" was also significantly high for one-person household group. This 
is the same as the result of the findings of Jung ${ }^{11)}$ that subjective health, stress, and subjective oral health had a higher proportion of elderly people living alone saying it is bad when compared to those living with family members. Moreover, the proportion of one-person households responding that they had a disease was significantly higher than that of multi-person households, which is consistent with the findings of Kim. ${ }^{10)}$ In conclusion, one-person households in the elderly population assessed that the subjective and objective health levels were less than the multi-person households.

Sleeping time was significantly lower in one-person households than in multi-person households, which is consistent with the finding of Han et al. ${ }^{14)}$ that elderly people living alone sleep 6.05 hours a day, less than 6.59 hours a day for couple elderly. This is because most of the elderly people living alone lack the economic and social support given by the cohabiting families, thus become easily neglected to behaviors to maintain the health of the mind and body, and consequently, this leads to deterioration of physical health or social psychological isolation. ${ }^{22)}$

As an indicator of the medical needs in terms of mental health, "suicide experience" and "suicide attempt experience" were of a higher proportion in one-person households than multi-person households. This is consistent with a study reported by Park et al. ${ }^{13)}$ that with oneperson households, women, low income and lower educational background, the higher the suicidal thoughts. This study assumed that there was a problem with mental health and one required mental health management and medical requirement in the case of "suicide experience" and "attempting suicide." These indicators were used as substitute indicators suggesting "needs for mental health services." The logical rationale for this approach starts from the claim that "suicide attempts" are medical conditions to be treated with behaviors in unhealthy conditions, including disease such as mental illness, and preventable medical and health problems. ${ }^{24)} \mathrm{Bae}$ and $\mathrm{Woo}^{25)}$ pointed out that intentions to commit suicide or attempts to commit suicide frequently lead to suicide; hence, they are signs of suicide and require preventive management and medical measures. In particular, mental health problems such as depression and stress have been proposed as direct factors for suicide of the elderly, ${ }^{26)}$ while these mental health problems are frequently encountered in the field where primary healthcare is provided, and must be emphasized as an issue that primary healthcare doctors should pay particular attention to. ${ }^{27)}$ Through empirical studies, Luoma et al. ${ }^{28)}$ corroborated that $90 \%$ of people who died from suicide used primary healthcare a year before death, and $76 \%$ visited a primary healthcare institution within 1 month of suicide. They suggested that addressing mental health and suicidal intention as a sign of suicide should be an important concern of primary healthcare doctors. As the elderly living alone are vulnerable to mental health conditions compared to multi-person households, and there are no other household members to assess suicidal signs, it is necessary to pay special attention to the mental health and suicidal signs of singleperson elderly households in the primary healthcare field.

As an indicator of medical accessibility, the percentage of not receiv- ing "oral examination," "health examinations," and "cancer examinations" was higher in one-person households than in multi-person households. The proportion of not receiving "dental care" and "needed medical care" experiences was also higher among one-person households. This is similar to the finding of $\mathrm{Kim}^{21)}$ that the rate of health examination and cancer examinations of the elderly living with their spouses is higher than that of the other group. These findings indicate that elderly people living alone have less access to medical care and less information than those living together. The recognition of the care service in the country is also attributed to the low result. However, as for the influenza immunization rate, one-person households were significantly higher than multi-person households, and a detailed analysis was conducted to find out the reason. Since there are more health centers than single hospitals for one-person household inoculation sites, it is possible that health institutions including public health centers have actively prevented the residents of rural areas, but further clarification is needed.

Logistic regression analysis was performed by clustering the various variables into groups of variables with the same meaning, and as a result, even though the age and gender were included in the model and controlled, the one-person household consumed 0.94 times less "bland food" than the multi-person household. The "nutrient labelling perception" also had 1.55 times higher "no perception" proportions compared to "perception." The disease score was calculated by coding 1 for the cases of diagnosis of four diseases; hypertension, diabetes, arthritis, and cataract. The higher the disease score, the greater the number of diseases. The one-person household group had a "combined disease score" of 1.02 times higher than the multi-person household group. This is similar to Kim and Kim, ${ }^{16)}$ who reported that elderly people living alone had more than three diagnosed disease compared to those not living alone and the research of Sohn ${ }^{17)}$ that found the chronic disease burden was 7.7 points for the elderly living alone and 4.8 points for the elderly not living alone. As such, most of the previous studies related to diseases were analyzed based on the presence of chronic disease, depression, and quality of life. There was no previous study using disease scores, so the above results could not be compared with other studies.

Sleeping time was shorter in one-person households than in multiperson households. Awareness of health and awareness of oral health were significantly worse, and awareness of depression was high. These results were similar to those of Jung ${ }^{11)}$ where there is a high percentage of elderly living alone with bad subjective health and subjective oral health. It also supports the research of Ryu and Park, ${ }^{29)}$ which claims that family is one of the most important factors affecting health status. Therefore, it is necessary to induce and improve the environment that elderly live in with their families rather than to stand alone. ${ }^{11)}$ "Mental health needs" was also 1.24 times higher, and these results indicate that the elderly living alone had a higher suicidal thought rate than elderly couples, as corroborated by Han et al. ${ }^{14)}$ It also showed similar results as the research of Jeon and Lee ${ }^{15)}$ and Kim, ${ }^{30)}$ which showed that suicidal thought experience is higher for those without a spouse. 
The "level of not receiving required services related to oral" was 1.03 times higher for one-person households. This was similar to the result of Jung, ${ }^{11)}$ which showed that the percentage of elderly who lived alone was 0.33 times less than the elderly who received oral examination. The "level of not receiving health examinations and medical use service" was 1.17 times higher for one-person households than the multiperson households.

In conclusion, one-person households are considered to have poorer overall health behaviors, such as nutritional behaviors, exercise behaviors, drinking and smoking behaviors, and poorer subjective and objective health levels than multi-person households. Furthermore, albeit the high requirement for mental health and medical services, such as suicide attempts, the high rate of not receiving services indicated high barriers to receive and access health care. The implications from these results are as follows. First, a variety of programs are needed to prevent health problems and improve health behaviors through appropriate education and training for elderly one-person households. Second, along with the high medical service needs, policy efforts are needed to profoundly investigate obstacles hindering actual medical use and to solve these obstacles. In particular, since the demand is not being properly connected to medical use compared to high needs of primary healthcare, it is necessary to devise institutional measures to enable continuous medical care for the single-person elderly households in the primary healthcare.

The purpose of this study was to analyze the socio-demographic characteristics, health behaviors and health status, medical needs and accessibility of Koreans in the one-person and multi-person households. Although the data were analyzed using Korean representative data, there are some limitations. First, since this is a cross-sectional study that examined health level, medical access, etc. at the time of survey in 2017, there is a limitation that it is not possible to causally interpret the difference between one-person and multi-person households. Second, recall bias may occur because the survey is a 1:1 interview. Third, there are limitations in applying stratification variables, cluster variables, and weight variables in research and analysis, thus although it is a study of all citizens, it is not a perfect representation of the entire population. Fourth, there is a small percentage of households in the one-person household, so there is a difference in the number of subjects between the comparison group and the control group. Hence, in statistical analysis, there is a possibility of underestimation and false negatives in some items.

Despite the above limitations, this study is meaningful in the following aspects. First, it is easy to generalize and apply the results to all citizens by utilizing the data of Community Health Surveys that have undergone strict quality control. Second, unlike previous studies analyzing mental health and quality of life of the elderly, the differences in characteristics were derived by directly comparing the health behaviors and health and medical factors between one-person and multiperson households. Comprehensive and direct comparison of health and medical factors revealed the medical vulnerability and characteristics of the single-person elderly households. Therefore, as a risk group, they revealed the unsatisfied medical needs to be improved for these single-person elderly households. Such data can be applied as basic information to understand these groups for primary medical doctors who predominantly treat single-person elderly households and can also be used as basic data for policy development in order to solve unsatisfied medical care problems.

One-person elderly households are the overlapping areas of two social challenges, namely, the increase in the proportion of one-person households and the expansion of the elderly population. They are considered as the most vulnerable class and this research corroborates such evidence succinctly. In particular, the increasing problem of elderly medical expenses is considered to be the first priority to solve the problem of sustainable health insurance financing. Preventive efforts concerning single-person households and diverse policy efforts to minimize obstacles to the use of medical services should be urgently devised. In particular, considering the characteristics of medical necessities of single-person elderly households, as sustainable medical care is important, policy incentives are required to take interest in these groups and effectively manage these groups in primary healthcare fields.

\section{CONFLICT OF INTEREST}

No potential conflict of interest relevant to this article was reported.

\section{ORCID}

Su-Jung Lee: https://orcid.org/0000-0003-3351-2937

Sun-Hee Lee: https://orcid.org/0000-0002-3628-7627

\section{REFERENCES}

1. Cho YK, Shim KW, Suk HW, Lee HS, Lee SW, Byun AR, et al. Differences between one-person and multi-person households on socioeconomic status, health behavior, and metabolic syndrome across gender and age groups. Korean J Fam Pract 2019;9:373-82.

2. Statistics Korea. Household projections 2010-2035 [Internet]. Daejeon: Statistics Korea; 2012 [cited 2019 Dec 16]. Available from: http://kostat. go.kr/portal/korea/kor_nw/1/2/1/index.board?bmode=read\&aSeq= 255176\&pageNo $=27 \&$ rowNum $=10 \&$ amSeq $=\&$ sTarget $=\&$ sTxt $=$.

3. Jung KH, Nam SH, Jung EJ, Lee JH, Lee YK, Kim JS, et al. Policy implications of changes in family structure: focused on the increase of single person households in Korea. Sejong: Korea Institute for Health and Social Affairs; 2012.

4. Kindicator. Ratio of elderly people [Internet]. Daejeon: Statistics Korea; 2020 [cited 2020 Jan 6]. Available from: https://www.index.go.kr/unify/idx-info.do?idxCd=8039.

5. Park JS, Yu YJ. Convergence factors influencing the quality of life in the elderly people living alone. J Digit Converg 2016;14:63-70.

6. Moon JH, Kim D. Factors influencing life satisfaction in elderly living alone. J Korea Contents Assoc 2018;18:44-54.

7. Shin SS, Choi KH, Kim YH, Park JH. Welfare costs of 6 trillion won for 
the people born in 1955..."The elderly medical expenses are bombs.” Joongangilbo [Internet]. 2020 Jan 6 [cited 2020 Jan 6]. Available from: https://news.joins.com/article/23674556.

8. Jang JS, Hong MS. A study on health-related lifestyle, dietary habits, nutritional knowledge and food intake of the elder in Gyeong-gi area. Korean J Food Nutr 2015;28:1056-64.

9. Kang HC. A study on comparison of Yangseng (traditional health behavior), depression, anxiety and cognitive function between the elderly women living alone and the aged in the living with family. J Orient Neuropsychiatry 2014;25:123-32.

10. Kim KS. Effects of the health status and health behavior on health-related quality of life of the elderly living alone and living with their families: using data from the 2014 Community Health Survey. J Korean Acad Community Health Nurs 2017;28:78-87.

11. Jung ES. Research on health and oral health status of elderly living alone compared to elderly living with their families: based on the data (2014) from the 6th two-year Korea National Health and Nutrition Examination Survey. J Korean Soc Dent Hyg 2017;17:99-110.

12. Sok SH. A comparative study on physical health status, family support, and life satisfaction between the aged living alone and living with family. J Korean Acad Community Health Nurs 2008;19:564-74.

13. Park BY, Kwon HJ, Ha MN, Burm EA. A comparative study on mental health between elderly living alone and elderly couples: focus on gender and demographic characteristics. J Korean Public Health Nurs 2016;30:195-205.

14. Han SS, Kang SW, Jung SH. Experiences of depressive symptom and suicidal thinking between the elderly living alone and the elderly couples. Korean J Health Serv Manag 2012;6:29-38.

15. Jeon GS, Lee HY. Impact of marital status on depression and suicidal ideation among Korean adults: focused on the difference by age and sex. Korean J Health Serv Manag 2011;5:179-90.

16. Kim HR, Kim EH. Prevalence of depression and its risk factors between elderly living alone and elderly living with others in Korea. J Korean Data Anal Soc 2014;16:463-77.

17. Sohn JN. A study on factors influencing the suicidal ideation in elderly people who live alone or live with family. J Korean Acad Psychiatr
Ment Health Nurs 2012;21:118-26.

18. Kim YJ. Comparison of health habits, perceived stress, depression, and suicidal thinking by gender between elders living alone and those living with others. J Korean Acad Fundam Nurs 2009;16:333-44.

19. Korea Centers for Disease Control and Prevention. Community Health Survey. Cheongju: Korea Centers for Disease Control and Prevention, Ministry of Health and Welfare; 2017.

20. Lee H, Cho YT. Comparison of health behaviors, disease prevalence between middle aged one-person households and multi-member households in South Korea. Health Soc Welf Rev 2019;39:380-407.

21. Kim JG. The impact of family type on health behavior of elderly people. Korean J Gerontol Soc Welf 2011;51:35-55.

22. Kim YS, Ha WY. A study of the effect of participation in productive leisure activities on the suicide ideation and physical and mental health of elderly living alone. Health Soc Welf Rev 2015;35:344-74.

23. Moon JH, Kang Ma. The prevalence and predictors of unmet medical needs among the elderly living alone in Korea: an application of the behavioral model for vulnerable populations. Health Soc Welf Rev 2016;36:480-510.

24. Rihmer Z. Strategies of suicide prevention: focus on health care. J Affect Disord 1996;39:83-91.

25. Bae SB, Woo JM. Suicide prevention strategies from medical perspective. J Korean Med Assoc 2011;54:386-91.

26. Jung MH, Heo SH. Study on the effects of suicide loss experience on suicidal ideation, plan and attempt of the elderly. Korean J Gerontol Soc Welf 2017;72:305-33.

27. Lee JH, Yang YJ. The role of primary care physicians to reduce suicide. Korean J Fam Pract 2013;3:240-4.

28. Luoma JB, Martin CE, Pearson JL. Contact with mental health and primary care providers before suicide: a review of the evidence. Am J Psychiatry 2002;159:909-16.

29. Ryu KS, Park HS. Comparison of health status between senior people living alone and those who live with their families. J Korea Gerontol Soc 2003;23:163-79.

30. Kim HK. Living alone of older persons and policy implications. Sejong: Korea Institute for Health and Social Affairs; 2011. 